\title{
SOFTWARE COMPARISON FOR UNDERWATER ARCHAEOLOGICAL PHOTOGRAMMETRIC APPLICATIONS
}

\author{
M. Vlachos ${ }^{a^{*}}$, L. Berger $^{\text {b }}$, R. Mathelier ${ }^{\text {b }}$, P. Agrafiotis ${ }^{\text {a }}$ and D. Skarlatos ${ }^{\text {a, }}$ \\ a 1 Cyprus University of Technology, Dep. Of Civil Engineering and Geomatics, P.O. Box 50329, Limassol 3603, Cyprus - \\ (marinos.vlachos. panagiotis,agrafioti, dimitrios.skarlatos)@cut.ac.cy \\ ${ }^{\mathrm{b}}$ National School of Geographic Sciences (ENSG), France - (louise.berger, rose.mathelier)@ensg.eu
}

\author{
Commission II, WG II/8
}

KEY WORDS: Underwater 3D Reconstruction, Underwater Photogrammetry, SfM MVS

\begin{abstract}
:
This paper presents an investigation as to whether and how the selection of the SfM-MVS software affects the 3D reconstruction of submerged archaeological sites. Specifically, Agisoft Photoscan, VisualSFM, SURE, 3D Zephyr and Reality Capture software were used and evaluated according to their performance in 3D reconstruction using specific metrics over the reconstructed underwater scenes. It must be clarified that the scope of this study is not to evaluate specific algorithms or steps that the various software use, but to evaluate the final results and specifically the generated 3D point clouds. To address the above research issues, a dataset from the ancient shipwreck, laying at 45 meters below sea level, is used. The dataset is composed of 19 images having very small camera to object distance ( 1 meter), and 42 images with higher camera to object distance ( 3 meters) images. Using a common bundle adjustment for all 61 images, a reference point cloud resulted from the lower dataset is used to compare it with the point clouds of the higher dataset generated using the different photogrammetric packages. Following that, a comparison regarding the number of total points, cloud to cloud distances, surface roughness, surface density and a combined 3D metric was done to evaluate and see which one performed the best.
\end{abstract}

\section{INTRODUCTION}

Underwater 3D modelling and mapping techniques are based on various systems and methodologies, but most recently the focus is on Structure from Motion (SfM) and multi View Stereo (MVS) techniques, which are based on RGB imagery as raw data (Menna et al., 2018). However, despite the relative low cost of these methods in relation to others, they present a major drawback; optical properties and illumination conditions of water severely affect underwater images (Agrafiotis et al., 2018a). Colours are absorbed at different rate as depth increases, resulting in a greenblue image, as light absorption mostly affects the red wavelengths. Water also absorbs light energy and scatters the optical rays thus creating blurred images.

The rise of fully automatic photogrammetric packages has helped archaeologists and photogrammetrists to fully record and reconstruct underwater archaeological finds with fine detail, which was almost impossible prior to commercial software using SfM and MVS algorithms. This also helped to the progress of underwater archaeological excavation as photogrammetrists are now able to provide updated results of the excavated sites and their changes in a daily basis. This revolution led to the speedup of the archaeological underwater excavation process, since archaeologists are provided with the full 3D reconstructed scenery every day for planning and organizing further steps in a faster pace.

However, since the commercial software market expands, this study tries to address which one of several available photogrammetric packages can provide better overall results on the point clouds of an underwater archaeological environment. To that direction, an underwater dataset at 45 meters below sea level is used. The dataset is composed of 19 images having very small camera to object distance ( 1 meter), and 42 images having larger camera to object distance (3 meters) images, of which the former used as reference to the latter data set.

\subsection{The Dataset and the Site's back story}

The dataset studied here has been captured in Mazotos shipwreck (Demesticha, 2011, Demesticha et al. 2014), at 45 meters below sea level, a depth where divers can work. It is assumed to be a 4th century BC commercial ship which sank near the village of Mazotos, located along the Southern coast of Cyprus. The images were acquired with artificial lighting due to the depth of $45 \mathrm{~m}$, using a Canon PowerShot A620 camera.

The shipwreck was reported 2007, with the first excavation period contacted during 2008, led by the Archeological Research Unit of the University of Cyprus, in collaboration with THETIS Foundation and the Department of Antiquities of Cyprus. Since 2010, the Department of Civil Engineering and Geomatics of the Cyprus University of Technology is responsible for 3D modelling and mapping the whole site, using photogrammetry; a method able to obtain rapid measurement with high accuracy (Demesticha et al., 2014).

\subsection{The Role of Photogrammetry in an UW Excavation}

Underwater photogrammetric data are acquired during excavation seasons in the case of Mazotos shipwreck (Demesticha et al., 2014). As the underwaterarchaeologists excavate one or several trenches of the wreck every day, one or two dives are devoted to photogrammetry. What the archaeologists expect from the photogrammetric survey is to provide in a daily basis, precise georeferenced data to monitor the site and localize the artefacts they wish recover. Working in a

\footnotetext{
*marinos.vlachos@cut.ac.cy; www.photogrammetric-vision.weebly.com.
} 
constantly changing environment is quite challenging for photogrammetry, as the acquisition must be carried out daily immediately after the excavation. If the acquisition stage is not sufficient, more artefacts will be removed the next day and the information will be lost without being documented.

The purpose of photogrammetry in an excavation is twofold; high quality georeferenced dense clouds, meshes and orthophotos of the whole wreck and of the trench currently excavated are needed for further studies, to have a record of the exact location and disposition of the finds. However, archaeologists also need orthophotos every day, to have an overview of the excavation site and to be able to plan and prepare the next steps. Since a highquality process take a long time to run, a faster low-quality process is what is done usually before the proper one by reducing the resolution of images by 16 (medium quality) or 32 (low quality) times, where for high quality the resolution of images is reduced by 4 times. This way the archaeologists can have all the necessary data like 3D textured models and orthophotos in time.

\section{PREVIOUS WORK}

Various works exist in the literature for comparing 3D data of different sources. However, the majority of those are dealing with over water data where control datasets are easier to be collected. Skarlatos and Kiparissi, (2012) tested Menci's Zscan BundlerPMVS and compared their results against a $300 \mathrm{~mm}$ diameter sphere with texture, a building façade and an electricity station. The image based techniques were then compared with two TLS stations in an effort to address the debate of accuracy, density, methodology and ease to use. Regarding the point cloud comparisons, De Asís López et al., (2014) applied standard deviation estimation to their measurements to evaluate the uncertainty of two point clouds of the same object, obtained with different equipment or in different conditions. Following that they adjusted to each of the point clouds a surface by means of a kernel smoothing technique and compared them.

A similar study as the one presented in this paper was performed by Schwind (2016) where the author compared and characterized the point clouds generated by Pix4D, Agisoft PhotoScan, and OpenDroneMap using the same dataset of UAV images. The characteristics that were directly compared among the point clouds were the number of key point matches, point density, point spacing, the number of points, and standard statistics such as minimum, maximum, mean, and standard deviation of the $\mathrm{X}, \mathrm{Y}$, and $\mathrm{Z}$ coordinates. Alidoost and Arefi (2017) investigated the capability of four different photogrammetric packages; 3DSurvey, Agisoft Photoscan, Pix4Dmapper Pro and SURE, in order to generate high density point clouds as well as a Digital Surface Models (DSM) over a historical site. Following that the authors performed absolute and relative point cloud assessments where for the former they evaluated the performance of dense point cloud generation, the number of key points per image, the number of final dense points, point count in a same extent, point spacing, spatial error for horizontal and vertical components, and the computational time. For the latter, they calculated the mean error and standard deviation of 3D distances between point clouds based on a six parameters quadric function.

Another relatively recent publication deals with the subjective quality assessment and metrics of point cloud geometry, subject to typical types of impairments such as noise corruption and compression-like distortions (Alexiou and Ebrahimi, 2017). More specifically the authors propose a subjective methodology that is closer to real-life scenarios of point cloud visualization. As it is stated by the authors, the performance of objective metrics is assessed by considering the subjective scores as the ground truth.

Finally, Mangeruga et al. (2018) selected five well-known methods from the state of the art and used them to enhance a dataset of images produced in various underwater sites with different conditions of depth, turbidity, and lighting. Part of their evaluation was done based on the results of $3 \mathrm{D}$ reconstructions.

\section{DATASETS AND METHODOLOGY}

Due to the considerable water depth, these images were acquired with artificial lighting, using a Canon PowerShot A620 camera, having resolution of $3072 \times 2304$ pixels and pixel size of $2.35 \times$ $2.35 \mu \mathrm{m}$. Moreover, 9 control points were used measured with the methodology described in Skarlatos et al., (2017) and Skarlatos et al., (2019). In the tests performed, the results of the first dataset of the 19 close range images was used as ground truth while the second one having 42 images (Figure 1) was used for evaluating software performance. Dense 3D point clouds were generated from the two datasets using the five well known SfMMVS software; Agisoft Photoscan, Reality Capture, 3D Zephyr, Visual SFM and SURE. It must be said that both reference and test datasets were combined in a single bundle block adjustment, and the 3D point clouds were generated separately. This approach was selected in order to avoid or minimize discrepancies from reference in a common coordinate system. In the end it was decided that only one point cloud would be used as a reference for the comparisons. The point cloud that was selected as reference was the one generated from the lower dataset in Photoscan. The reason Photoscan was chosen is that it is being used for underwater photogrammetry (Demesticha et al., 2014), so it is known to produce fairly good results in this underwater environment. Although just from that selection alone it seems that the final results will heavily favourite the Photoscan dense cloud, that is not the case as it is shown in section 5. The produced 3D point clouds were compared using Cloud Compare (Cloud Compare, 2019) open-source software and statistical measures between reference and each of the point clouds resulted from the different software used were computed. The followed process is quite similar to the one presented in Agrafiotis et al. (2017), Agrafiotis et al. (2018) and Mangeruga et al. (2018). In particular, the following parameters and statistics were computed for each point cloud:

- Total number of points.

- Cloud to cloud distances. Cloud to cloud distances are computed by selecting two-point clouds. The default way to compute this kind of distance is the "nearest neighbour distance': for each point of the compared cloud, Cloud Compare searches the nearest point in the reference cloud and computes the Euclidean distance between them (Cloud Compare, 2019).

- $\quad$ Surface Density. The density is estimated by counting the number of neighbours $\mathrm{N}$ (inside a sphere of radius R) for each point. The surface density used for this evaluation is defined the number of neighbours divided by the neighbourhood surface. Cloud Compare estimates the surface density for all the points of the cloud and then it calculates the average value for an area of $1 \mathrm{~m}^{2}$ in a proportional way. Surface density is considered to be a positive metric, since it defines the number of the points on a potential generated surface, excluding the noise being present as points out of this surface. This is also the reason of using the surface density metric instead of the volume density metric. 
- Roughness. For each point, the 'roughness' value is equal to the distance between this point and the best fitting plane computed on its nearest neighbours (Cloud Compare, 2019), which are the points within a sphere centred on the point. Roughness is considered to be a negative metric since it is an indication of noise on the point cloud, assuming an overall smooth surface.

To facilitate an overall comparison of the tested software in terms of $3 \mathrm{D}$ reconstruction performance and evaluate the numerous results, the surface density $D$ and roughness $R$ metrics were normalized and combined into one overall metric, the Combined 3D metric (Mangeruga et al., 2018). According to the objective, metrics and results retrieved are presented with a critical point of view, highlighting the pros and the cons of each software for the dataset used. Following that, an overall conclusion will be attempted on which of the five aforementioned software packages performs best in this particular environment and for this particular dataset.

\section{IMPLEMENTATION}

In this section the implementation of the study is showcased. It must be noticed here that among the metrics of comparison, the number of feature points on the images was not taken into account as the comparison is done exclusively on the point clouds the software packages generate. No pre-processing step was done to radiometrically correct the dataset as the study does not evaluate the impact of the radiometry in the final results.

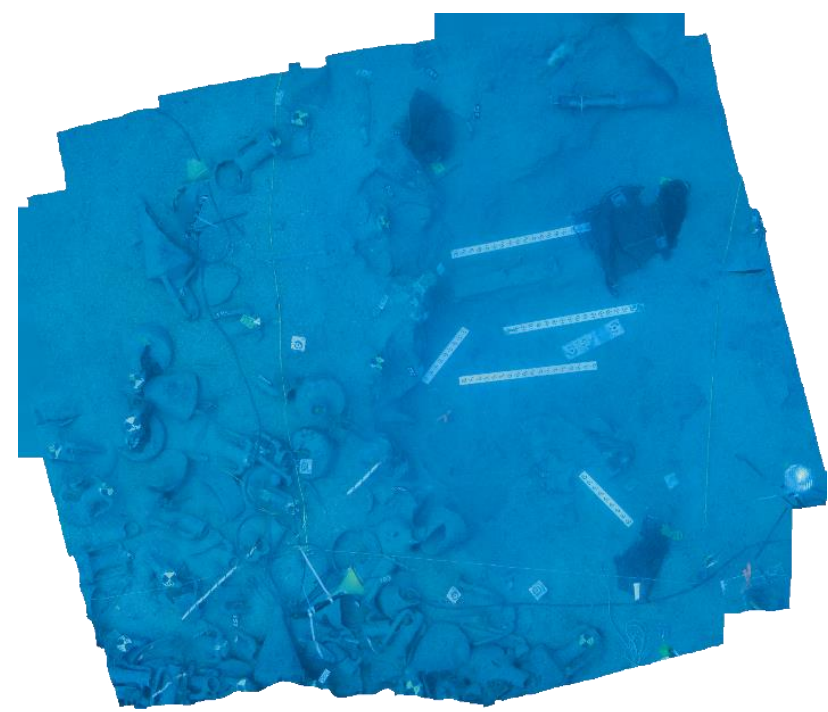

Figure 1: Orthophoto from original dataset without radiometric corrections.

To facilitate the comparison between all the dense clouds, a dense cloud is needed to serve as reference. This reference must have the highest possible quality thus the green channel was selected to extract this reference point cloud The use of the green channel is due to its better performance in a photogrammetric point of view (Mandlburger et al., 2018) and more specifically regarding the feature point extraction and matching. The alignment was done with the 61 images, in high quality. As said before the reference for all the comparisons was the high-quality dense cloud extracted from Photoscan using only the 19 lower images. For all software packages, the 42 other images of the high-level were used to generate the "test" dense clouds (Figure 2). All test point clouds were extracted from the green channel as well. The reason that for all point clouds the green channel was selected is because first of all, with that the effect of colour aberration is eliminated (Luhmann et al., 2006), an effect which is very noticeable in the UW imagery especially near the image borders. Another reason the green channel is selected for the $3 \mathrm{D}$ reconstruction is that in the depth of $45 \mathrm{~m}$ where the site is, even with the use of artificial lighting, the absorption of red is significant. For that reason and because the camera sensors record double the information in the green channel, this approach was selected.
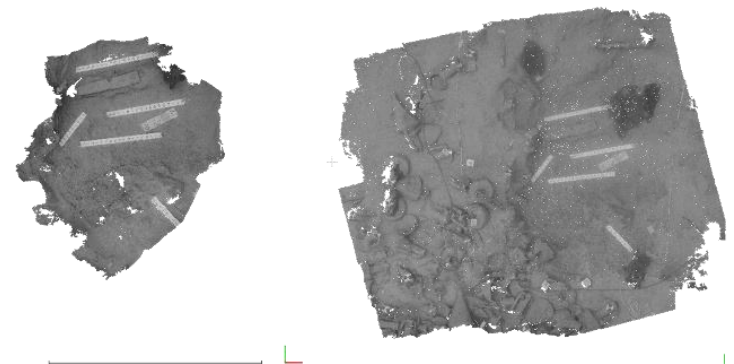

Figure 2: The Reference Point Cloud (Left) and The Photoscan tested point cloud from the higher dataset.

\subsection{Cloud to Cloud distances}

The absolute distances between two dense clouds are calculated with the "cloud to cloud distances" tool of CloudCompare. The compared cloud is the one on which distances will be computed. CloudCompare will compute the distances of each of its points relatively to the reference cloud (Figure 3 ). The reference cloud is the cloud that will be used as reference, i.e. the distances will be computed relatively to its points. If possible, this cloud should have the widest extents and the highest density. Each calculation provides the mean and the standard deviation that allows to compute the RMS (Root Mean Square). In fact, the definition of the mean and the standard deviation are respectively:

$$
\begin{gathered}
\mu=\frac{1}{\mathrm{~N}} \sum_{i=1}^{N} x_{i} \\
\sigma=\sqrt{\frac{1}{N} \sum_{i=1}^{N}\left(x_{i}-\mu\right)^{2}}
\end{gathered}
$$

The RMS is given by this formula:

$$
R M S=\sqrt{\frac{1}{N} \sum_{i=1}^{N} x_{i}{ }^{2}}
$$

It can be computed by the merge of the mean and the standard deviation with this equation:

$$
R M S=\sqrt{\mu^{2}+\sigma^{2}}
$$

\subsection{Roughness}

To calculate the roughness, the "roughness" tool of CloudCompare was used (Figure 4). For each point, the 'roughness' value is equal to the shortest distance between this point and the best fitting plane computed on its nearest neighbours (Cloud Compare, 2019). The kernel size chosen is $0.025 \mathrm{~m}$. Thus, the lower is the value, the less rough is the dense 
cloud. The value used for our comparisons is the mean of the roughness for all the point in the dense cloud.

\subsection{Surface Density}

In order to find the surface density of the model, the "density" tool is used with the setting "surface density" of CloudCompare. The surface density is the number of neighbours divided by the neighbourhood surface (Cloud Compare, 2019). In the example below, the radius equals to $0.025 \mathrm{~m}$ (Figure 5).

$$
\text { Surface Density }=\frac{N}{\pi r^{2}}
$$

The value used for the comparisons is the mean of the surface density for all the points in the dense cloud.

\section{COMPARISONS}

In most of the tested point clouds it was noticed that the overall visual presentation of the dense cloud was better with medium quality as it looked fuller and with less noise.

\begin{tabular}{ccccc}
\hline & $\begin{array}{c}\text { Number of } \\
\text { points in the } \\
\text { dense cloud }\end{array}$ & $\begin{array}{c}\text { Mean } \\
\text { Roughness( } \\
\mathrm{m})\end{array}$ & $\begin{array}{c}\text { Mean number } \\
\text { of neighbors for } \\
\text { each point with } \\
\mathrm{r}=0.025 \mathrm{~m}\end{array}$ & $\begin{array}{c}\mathrm{C} 2 \mathrm{C} \\
\text { distances } \\
\text { RMSE }(\mathrm{m})\end{array}$ \\
\hline Reference & $10.5 \mathrm{M}$ & 0.001865 & $3 \mathrm{M}$ & \\
PhotoScan & $2.5 \mathrm{M}$ & 0.002806 & $90 \mathrm{~K}$ & 0.02244 \\
3DZephyr & $320 \mathrm{~K}$ & 0.001874 & $20 \mathrm{~K}$ & 0.02433 \\
RealityCapture & $2.4 \mathrm{M}$ & 0.001558 & $98 \mathrm{~K}$ & 0.01447 \\
VSFM & $531 \mathrm{~K}$ & 0.003225 & $30 \mathrm{~K}$ & 0.01604 \\
Sure & $40 \mathrm{M}$ & 0.003713 & $2.2 \mathrm{M}$ & 0.02276 \\
\hline
\end{tabular}

Table 1: Metrics of comparisons between the point clouds the 5 different packages produce

As it is shown in Table 1, the number of generated points per cloud differs significantly. From what is shown, SURE produced by far the larger amount of points. On the other hand, Reality Capture and Photoscan produced almost the same amount of points (ca. 2.5 million) where VSFM and Zephyr produced far less $(531 \mathrm{~K}$ and $320 \mathrm{~K})$. That potentially indicates that the MVS algorithms and the parameters used in Reality Capture and Photoscan are very similar to each other as well as the ones that are used for VSFM and Zephyr.

Regarding the C2C RMS error comparisons is noticeable that the less absolute distance achieved by the point cloud generated by Reality Capture $(1.45 \mathrm{~cm})$ with the one of VSFM $(1.60 \mathrm{~cm})$ having the second best. Photoscan comes in third $(2.24 \mathrm{~cm})$ even though the reference point cloud is created by Photoscan. Following is the SURE point cloud $(2.28 \mathrm{~cm})$ and last the $3 \mathrm{D}$ Zephyr one $(2.43 \mathrm{~cm})$. Based on that, it is assumed that the Photoscan point cloud even though was expected to have the least RMS error it does not due to noise presence.

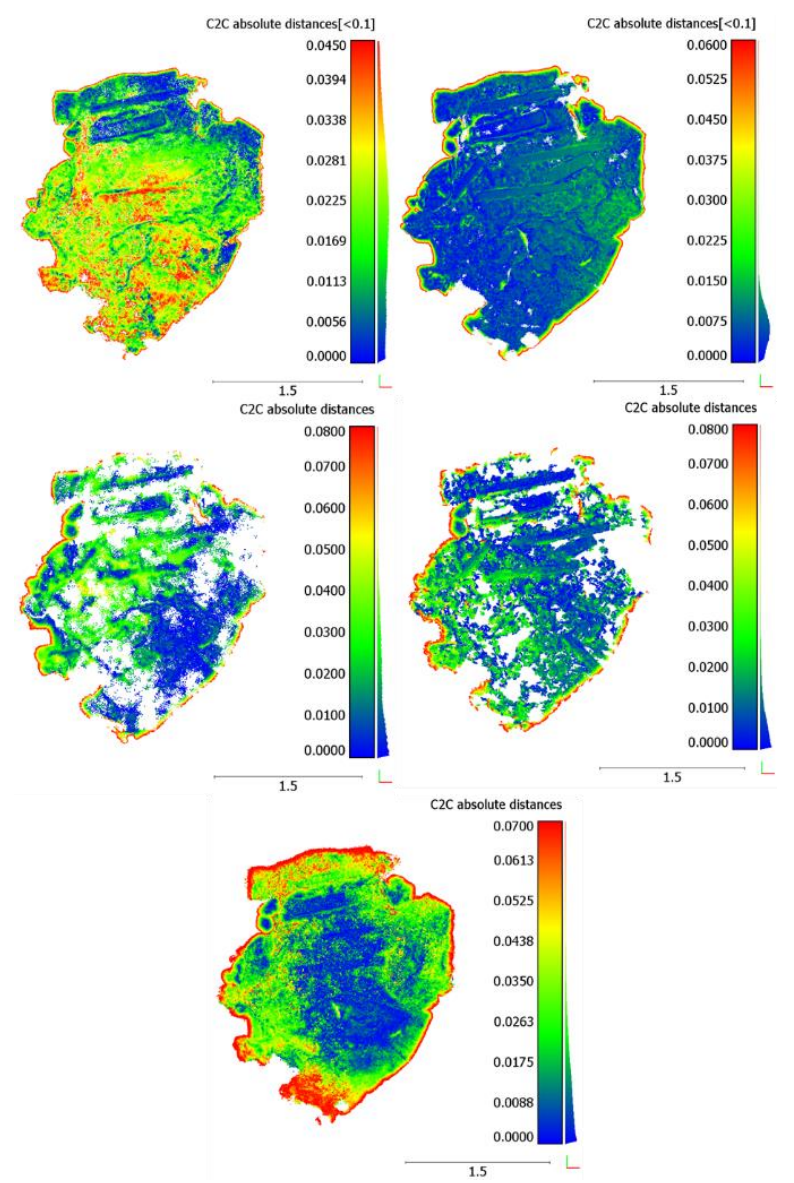

Figure 3: C2C Comparisons. Upper left: Photoscan, Upper right: Reality Capture, Middle Left: 3D Zephyr, Middle right: VSFM, Bottom: SURE

As it can be seen in Figure $4 \& 5$ the Cloud to Cloud statistics might be irrelevant when the derived point clouds do not have a good distribution of points and completeness like in the case of 3D Zephyr and VSFM.

Comparing the mean roughness of all the point clouds the assumption regarding the presence of noise in the Photoscan point cloud is validated with the mean roughness being at $2.81 \mathrm{~mm}$. A relatively high value compared to the Reality Capture which has the least amount of noise based on the mean roughness $(1.56 \mathrm{~mm})$ as it also goes along with the fact that it has the least RMS error in the C2C comparisons. Also, the 3D Zephyr dense point cloud appears to have a good mean roughness $(1.87 \mathrm{~mm})$. Finally, SURE and VSFM dense clouds have the larger mean roughness with $3.23 \mathrm{~mm}$ and $3.71 \mathrm{~mm}$ respectively. 


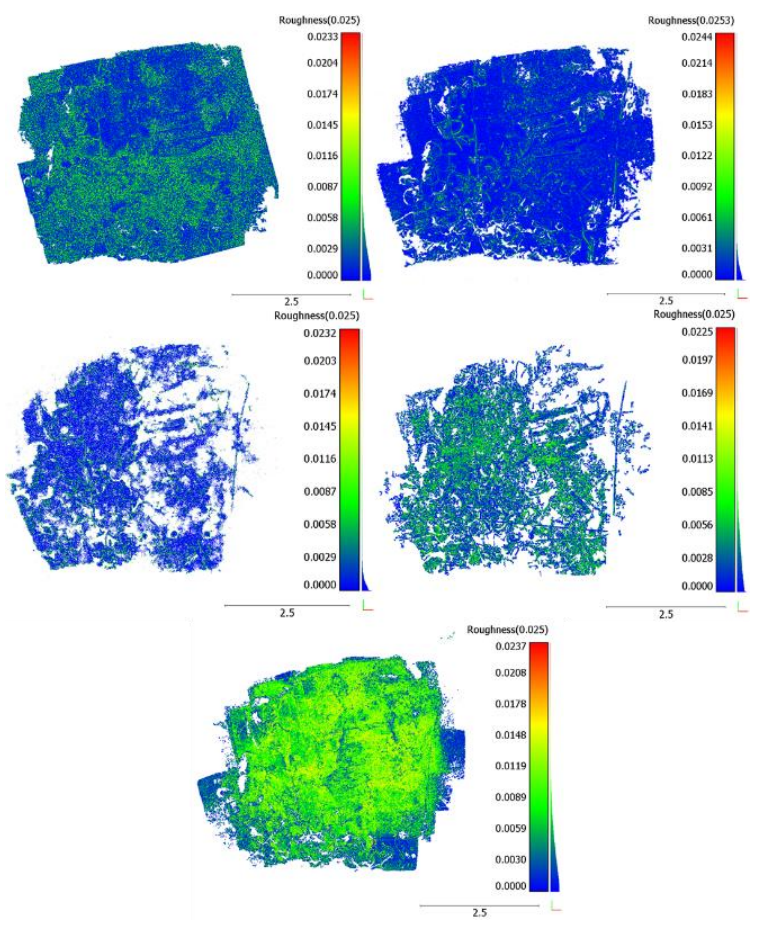

Figure 4: Roughness of all the points in the Dense Clouds. Upper left: Photoscan, Upper right: Reality Capture, Middle Left: 3D Zephyr, Middle right: VSFM, Bottom: SURE

Regarding the surface density as it is shown in Table 1, SURE has the biggest mean number of neighbours for every point with 2.2 million. Reality Capture and Photoscan have a mean density of $98 \mathrm{k}$ and $90 \mathrm{k}$ respectively where 3D Zephyr and VSFM have $20 \mathrm{k}$ and $30 \mathrm{k}$ respectively. Based on the number of points per dense cloud, the surface density metrics are nothing out of the ordinary.

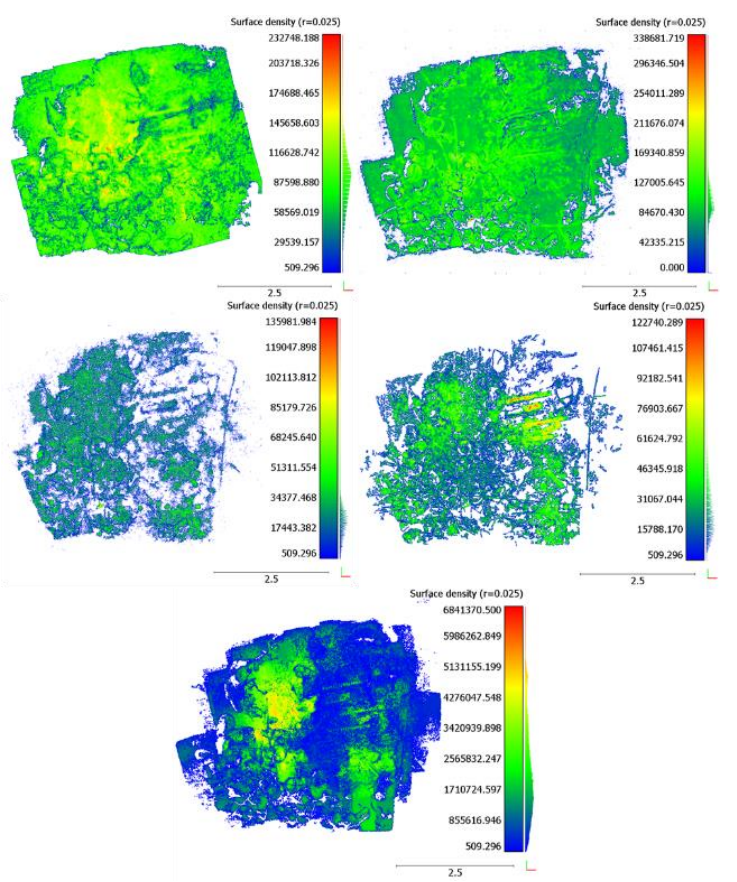

Figure 5: Surface Density across the Dense Clouds. Upper left: Photoscan, Upper right: Reality Capture, Middle Left: 3D Zephyr, Middle right: VSFM, Bottom: SURE

As mentioned before, to facilitate an overall comparison of the tested software in terms of $3 \mathrm{D}$ reconstruction performance and evaluate the results, the surface density $D$ and roughness $R$ metrics were normalized and combined into one overall metric, named as the Combined 3D metric. To achieve that, the score of every software on $\mathrm{D}$ and $\mathrm{R}$ was normalized to the score of reference dense cloud. Hence, the $100 \%$ score is referred to the reference dense cloud. If a software has a negative impact on the $3 \mathrm{D}$ reconstruction the score is less than $100 \%$.

$$
\begin{gathered}
\widehat{D}=\left|1-2 *\left(\frac{\text { Dsoft }}{\text { Dref }}\right)\right| \\
\hat{R}=\left|2-\left(\frac{\text { Dsoft }}{\text { Dref }}\right)\right|
\end{gathered}
$$

Due to the fact that roughness is a negative metric, the normalization equation differs from the one of surface density.

$$
\begin{gathered}
A v=\frac{\widehat{R}+\widehat{D}}{2} \\
C 3 D m=\frac{A v_{-} r e f}{A v_{-} s o f t}
\end{gathered}
$$

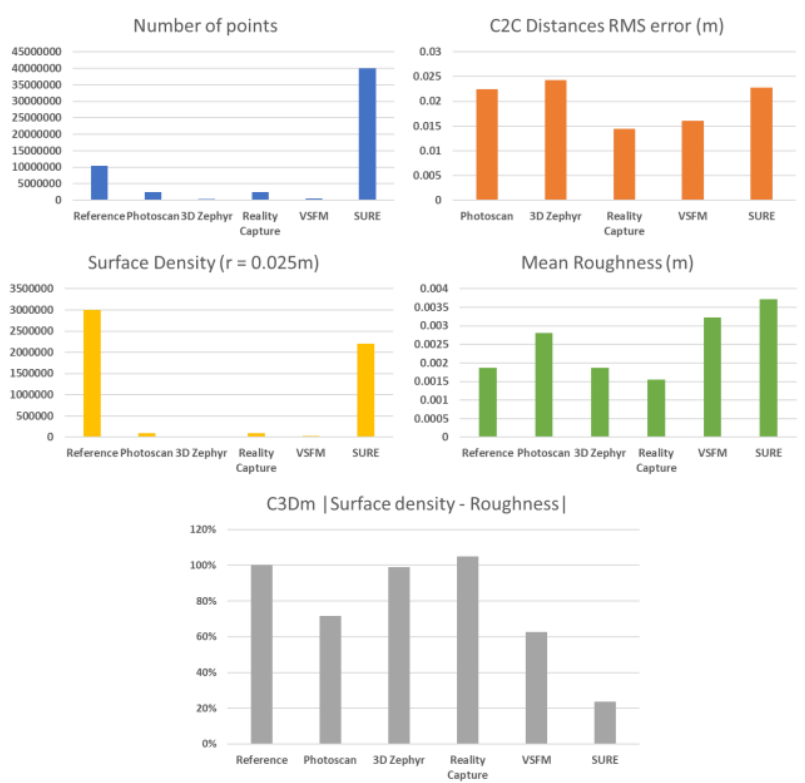

Figure 6: Visual representation of all the metrics that were evaluated

Comparing the overall $3 \mathrm{D}$ reconstruction performance of the packages the conclusion is that Reality Capture produces the most valuable results with 3D Zephyr being very close to it at $99 \%$. Next comes Photoscan with $72 \%$ due to the large value in roughness. Nevertheless, the results shown in Figure 6 and Table 2 must be interpreted very carefully. Indeed, just by observing the Combined 3D metric values someone immediately might consider Reality Capture and 3D Zephyr as the best options. For the former that assumption would not be incorrect but for the latter that would not be the case since the RMS error of the 3D Zephyr dense cloud is ignored as well as the completeness that is missing. 


\begin{tabular}{|c|c|c|c|c|c|c|c|}
\hline Name & Points & $\begin{array}{l}\text { C2C distances } \\
\text { RMSE }(\mathrm{m})\end{array}$ & $\begin{array}{l}\text { Surface } \\
\text { Density }\end{array}$ & $\begin{array}{c}\text { Mean } \\
\text { Roughness }(\mathrm{m})\end{array}$ & $\begin{array}{l}\text { Normalized } \\
\text { density to } \\
\text { the best }\end{array}$ & $\begin{array}{l}\text { Normalized } \\
\text { roughness } \\
\text { to the worst }\end{array}$ & $\begin{array}{c}\text { Combined 3D } \\
\text { metric (C3Dm) } \\
\text { |Surface density - } \\
\text { Roughness| }\end{array}$ \\
\hline Reference & 10500000 & & 3000000 & 0.001865 & $100 \%$ & $100 \%$ & $100 \%$ \\
\hline Photoscan & 2500000 & 0.02244 & 90000 & 0.002806 & $94 \%$ & $50 \%$ & $72 \%$ \\
\hline 3D Zephyr & 320000 & 0.02433 & 20000 & 0.001874 & $99 \%$ & $100 \%$ & $99 \%$ \\
\hline $\begin{array}{l}\text { Reality } \\
\text { Capture }\end{array}$ & 2400000 & 0.01447 & 98000 & 0.001558 & $93 \%$ & $116 \%$ & $105 \%$ \\
\hline VSFM & 531000 & 0.01604 & 30000 & 0.003225 & $98 \%$ & $27 \%$ & $63 \%$ \\
\hline SURE & 40000000 & 0.02276 & 2200000 & 0.003713 & $47 \%$ & $1 \%$ & $24 \%$ \\
\hline
\end{tabular}

Table 2: Combined 3D metric $(\mathrm{C} 3 \mathrm{Dm})$ of the surface density and Roughness of the point clouds the 5 different packages produce

\section{CONCLUSIONS}

Based on the evaluated metrics, one can conclude where each software thrives and falls. In the case of completeness, Photoscan, Reality Capture and SURE seem to produce more complete point clouds. SURE needs the exterior orientation and camera calibration parameters to be known from another software since it is not a standalone software. Having that in mind, one could say that SURE wouldn't be the option to produce daily 3D point clouds especially during an archaeological expedition where the photogrammetric processing needs to be fast.

From the metrics that were evaluated during this particular study it can be seen that Reality Capture and VSFM when compared to the reference have the lowest RMS errors with the Reality Capture point cloud being the best overall. Additionally, seeing the results regarding the roughness is noticeable that reality capture outperforms the other software. Unfortunately, a clear conclusion regarding the surface density metrics cannot be extracted due to the fact that the number of neighbour points is proportionate to the total number of points the point cloud has.

Taking everything into consideration regarding point cloud completeness, number of points, point distribution, and all the metrics that were evaluated it can be said that Reality Capture and Photoscan could be the best options for the production of 3D dense point clouds underwater. Additionally, these two packages can generate textured 3D models and orthophotos and combining them with their overall performance on the dense point cloud generation, they seem to be the most reliable options.

Even though from those comparisons it seems that some photogrammetric packages might have the edge over the others, we cannot rely only on the results of this particular dataset as the change of scenery, a dataset with better radiometric quality i.e. more presence of red in the images, the conditions underwater in a particular day and time as well as the depth of the UW site might affect the final result. For that reason, in the future more evaluations with different datasets under different conditions can give concrete conclusions about which photogrammetric package produces the best overall $3 \mathrm{D}$ point clouds in an underwater environment.

\section{ACKNOWLEDGEMENTS}

The contribution of M. Vlachos, P. Agrafiotis and D. Skarlatos is part of iMARECULTURE project (Advanced VR, iMmersive Serious Games and Augmented REality as Tools to Raise Awareness and Access to European Underwater CULTURal heritagE, Digital Heritage) that has received funding from the European Union's Horizon 2020 research and innovation programme under grant agreement No 727153 . The authors would like to thank Professor Stella Demesticha, director of the
Mazotos Shipwreck excavation, the department of antiquities of Cyprus for allowing the use of this particular dataset. Additionally, we would like to thank Markos Garras for the image acquisition.

\section{REFERENCES}

Agrafiotis, P., Drakonakis, G. I., Georgopoulos, A., and Skarlatos, D., 2017. The Effect of Underwater Imagery Radiometry on 3D Reconstruction and Orthoimagery. The International Archives of the Photogrammetry, Remote Sensing and Spatial Information Sciences, XLII-2/W3, 25-31, https://doi.org/10.5194/isprs-archives-XLII-2-W3-25-2017,

Agrafiotis, P., Drakonakis, G., Skarlatos, D., Georgopulos, A., 2018. Underwater Image Enhancement before ThreeDimensional (3D) Reconstruction and Orthoimage Production Steps: Is It Worth? Latest Developments in Reality-Based 3D Surveying and Modelling, 239-256. https://doi.org/10.3390/books978-3-03842-685-1-11

Agrafiotis, P., Skarlatos, D., Forbes, T., Poullis, C., Skamantzari, M., and Georgopoulos, A., 2018. Underwater Photogrammetry in Very Shallow Waters: Main Challenges and Caustics Effect Removal. The International Archives of the Photogrammetry, Remote Sensing and Spatial Information Sciences, XLII-2, 1522. https://doi.org/10.5194/isprs-archives-XLII-2-15-2018

Alexiou, E., Ebrahimi, T., 2017. On the Performance of Metrics to Predict Quality in Point Cloud Representations, in: SPIE Proceedings, 53. https://doi.org/10.1117/12.2275142

Alidoost, F., Arefi, H., 2017. Comparison Of UAS-Based Photogrammetry Software For 3D Point Cloud Generation: A Survey Over A Historical Site. ISPRS Annals of the Photogrammetry, Remote Sensing and Spatial Information Sciences, 4, 55-61. https://doi.org/10.5194/isprs-annals-IV-4W4-55-2017

CloudCompare (Version 2.10 alpha) [GPL Software]. 2019. Available online: http://www.cloudcompare.org/

De Asís López, F., Ordóñez, C., Roca-Pardiñas, J., GarcíaCortés, S., 2014. Point Cloud Comparison Under Uncertainty. Application to Beam Bridge Measurement with Terrestrial Laser Scanning. Journal of the International Measurement Confederation, $51, \quad 259-264$. https://doi.org/10.1016/j.measurement.2014.02.013

Demesticha, S., 2011. The 4th-Century-BC Mazotos Shipwreck, Cyprus: A preliminary report. International Journal of Nautical Archaeology, 40, 39-59. https://doi.org/10.1111/j.10959270.2010.00269.x 
Demesticha, S., Skarlatos, D., Neophytou, A., 2014. The 4thCentury B.C. shipwreck at Mazotos, Cyprus: New techniques and Methodologies in the 3D Mapping of Shipwreck Excavations. Journal of Field Archaeology, 39, 134-150. https://doi.org/10.1179/0093469014Z.00000000077

Luhmann, T., Hastedt, H., Tecklenburg, W., 2006. Modelling of Chromatic Aberration for High Precision Photogrammetry. ISPRS Commission V Symp. on Image Engineering and Vision Metrology, XXXVI, 173-178.

Mandlburger, G., Kremer, J., Steinbacher, F., Baran, R., 2018. Investigating the Use of Coastal Blue Imagery for Bathymetric Mapping of Inland Water Bodies. The International Archives of Photogrammetry and Remote Sensing Spatial Information Sciences. - ISPRS Archives, 42, 275-282. https://doi.org/10.5194/isprs-archives-XLII-1-275-2018

Mangeruga, M., Bruno, F., Cozza, M., Agrafiotis, P., \& Skarlatos, D., 2018. Guidelines for Underwater Image Enhancement Based on Benchmarking of Different Methods. Remote Sensing, 10(10), 1652.

Menna, F., Agrafiotis, P., \& Georgopoulos, A., 2018. State of the Art and Applications in Archaeological Underwater 3D Recording and Mapping. Journal of Cultural Heritage, 33, 231248. https://doi.org/10.1016/j.culher.2018.02.017

Schwind, M., 2016. Comparing and Characterizing ThreeDimensional Point Clouds Derived By Structure from Motion Photogrammetry. Texas A\&M University - Corpus Christi, Corpus Christi Texas.

Skarlatos, D., Agrafiotis, P., Menna, F., Nocerino, E., \& Remondino, F., 2017. Ground Control Networks for Underwater Photogrammetry in Archaeological Excavations. In Proceedings of the 3rd IMEKO International Conference on Metrology for Archaeology and Cultural Heritage, Lecce, Italy (pp. 23-25).

Skarlatos, D., Kiparissi, S., 2012. Comparison of Laser Scanning, Photogrammetry and SfM-MVS Pipeline Applied in Structures and Artificial Surfaces. ISPRS Annals of the Photogrammetry, Remote Sensing and Spatial Information Sciences, 1, 299-304. https://doi.org/10.5194/isprsannals-I-3-299-2012

Skarlatos, D., Menna, F., Nocerino, E., and Agrafiotis, P., 2019. Precision Potential of Underwater Networks for Archaeological Excavation Through Trilateration and Photogrammetry. The International Archives of the Photogrammetry, Remote Sensing and Spatial Information Sciences, XLII-2/W10, 175-180, https://doi.org/10.5194/isprs-archives-XLII-2-W10-175-2019 